\title{
Integrated Fuzzy based Decision Support System for the Management of Human Disease
}

\author{
Blessing Ekong ${ }^{1}$, Idara Ifiok ${ }^{2}$, Ifreke Udoeka ${ }^{3}$, James Anamfiok ${ }^{4}$ \\ Dept. of Computer Science, Akwa Ibom State University \\ Ikot Akpaden, Nigeria
}

\begin{abstract}
To eliminate some of the inaccuracies in the diagnosis of human diseases, decision support systems based on algorithms and technologies such as Artificial Neural Network, Fuzzy Logic etc. have been used. The results of such diagnosis are used for treatment and management purposes. Inaccurate and imprecise diagnosis may lead to wrong treatment methods which in turn may result in death or complications. Although treatments are widely carried out using drugs, there exist other treatment methods such as alternative medicine, complimentary medicine which could be used for treatment. We propose an Integrated Fuzzy Based Decision Support System which focuses on the integration of both alternative and pure medicine for the management of malaria. The results obtained showed that integrating these two treatment and management methods will eliminate the limitations of the individual methods therefore bridging the gap between alternative and pure medicine in the treatment and management of human diseases. The system is implemented in $\mathrm{CH}$.
\end{abstract}

Keywords-Human diseases; fuzzy based decision support system; human disease; fuzzy logic; C\#

\section{INTRODUCTION}

Inaccuracies and imprecision in the conventional method of diagnosis, treatment and management of human diseases (e.g. malaria), recent trends in technology and the demand to stay relevant in today's competitive world of Information Technology have compelled many in the medical field to adopt the computer- based method of diagnosis, treatment and management of diseases. Such systems operate on the principles of Artificial Intelligence (AI) [1] and are developed for both diagnosis and treatment of diseases. The intelligence of such system is based on the result of an interactive decision process that uses facts and rules to solve real life problems [2], based on knowledge obtained from one or more human expert in specific areas. This could be achieved using fuzzy logic which is a branch of AI.

Fuzzy Logic is a form of multi-valued logic derived from fuzzy set theory to deal with approximate reasoning [3]. It offers a suitable way of representing and processing linguistic information and subjective attributes of the real world, this is presented in the work of [4]. According to the work Fuzzy logic systems adopt a specific lifecycle model which could be divided into four different components or stages [5]; fuzzifier component, fuzzy inference engine, and defuzzifier component. Fuzzy based systems are built to handle complex and sophisticated tasks which may be ambiguous to handle otherwise.
Decision Support System (DSS) refers to a group of components (machine and/or human) working together to provide information for efficient and strategic decision making. Efficiency in this context includes the cost in terms of time and resources involved in implementing the option specified by the decision support system. Conventionally, DSS is seen as a software or collection of computer programs which process raw data to provide information for efficient and strategic decision making. In the medical field, the use of DSS reduces or eliminates inaccuracies in diagnosis and even treatment of diseases.

Diagnosis is not very useful if there is no associated treatment for the diagnosed illness. As such, most diagnosis systems are developed for both diagnosis and treatment. The result of the diagnosis phase determines the treatment recommended. Unfortunately, most of the treatments recommended by such systems are based purely on drugs i.e. pure medicine known as orthodox method. As good as this may be, it is not a one size fit all approach that works always at all times. There exists many non-orthodox treatment approaches such as herbalism which could be used for the treatment of diseases. Herbalism involves the use of herbs for the treatment of diseases. This could be used where drugs are not available or appropriate or complimentarily with other drugs.

In this work, an integrative fuzzy based approach for the diagnosis, treatment and management of diseases is proposed. Diagnosis is carried out using fuzzy logic, based on the outcome derived from the diagnosis phase, treatment or management could be recommended. Treatments are administered using drugs and/or herbs. The medical history of the patient is strictly considered in the treatment of diagnosed illnesses. Medical history contains information such as last treatment period, and details of other health conditions such as pregnancy, ulcer etc. which may interfere with some drugs or herbs. Management in the context of this work involves preventive measures such as anti-malaria drugs, changes in lifestyle such as the use of mosquito nets for the prevention or control of diseases.

\section{FUZZY LOGIC}

Fuzzy Logic (FL) incorporates a simple, rule-based such as IF (condition) AND (condition) THEN (action) method to handle problems instead of the use of mathematical model. It provides a precise approach which could be used to make conclusion using imprecise assumptions. FL is a form of manyvalued logic or probabilistic logic; it deals with reasoning that 
is approximate instead of fixed and exact [6]. It is similar in many ways to Fuzzy sets. A fuzzy set B in Y is expressed as a set of ordered pairs:

$\mathrm{B}=\{(\mathrm{y}, \mu \mathrm{B}(\mathrm{y})) \mid \mathrm{y} \epsilon \mathrm{Y}\}$

$\mu \mathrm{B}: \mathrm{Y} \rightarrow \mathrm{N}$

Where: $\mu \mathrm{B}$ is the membership function.

$\mathrm{N}$ is the membership space and each element of $\mathrm{Y}$ is mapped to $\mathrm{N}$ such that if $\mathrm{N}=\{0,1\}, \mathrm{B}$ is a crisp set. Notwithstanding, if $\{0 \leq N \geq 1\}$, B is a fuzzy set.

\section{Management of Malaria}

Disease management refers to the concept of reducing health care costs and improving quality of life for individuals with chronic conditions by preventing or minimizing the effects of the disease through integrated care [7].Some of the activities involved in disease management include; prevention, diagnosis, treatment, education.

Prevention: According to the old saying that prevention is better than cure, the cost of preventing malaria is usually lower than the cost of treatment. Although some of the drugs used for the treatment of malaria may also be taken for prevention, Malaria could be prevented in the following ways;

Medication: This involves the use of drugs such as doxycycline, chloroquine, etc. for the prevention of malaria. This method of prevention is also known as chemoprophylaxis. The limitations in this method include the fact that some of these drugs have not been tested for long-term use, are not without side effect and may also be costly. Notwithstanding, anti-malarial drugs can be administered in a particular way to people at high-risk for malaria, such as pregnant women and infants $[8]^{1}$. Here, such people are given a dose(s) of antimalaria drugs for prevention. This is referred to as Intermittent Preventive Therapy (IPT).

Vector Prevention: This implies prevention without the use of medications such as; the use of insecticide treated mosquito net, adequate environmental hygiene, the use of long-clothing and insect repellent in the evenings and at night [8], when mosquitoes are very active. The number of mosquitoes can also be reduced by the use of chemical substance known as insecticide and spraying of repellents indoors.

\section{A. Orthodox Medicine Diagnosis and Management Approach}

Conventionally, blood tests can show the presence of the parasite and help tailor treatment by determining the present of malaria infection, type of malaria causative parasite, and the organs affected [9] ${ }^{2}$. According to the 2017 world malaria report by World Health Organization, antimalarial drugs should only be administered to individuals with suspected malaria case after they have been subjected to parasitological

\footnotetext{
${ }^{1}$ Malaria diagnosis and treatment-Mayo Clinic.Available at

https://www.mayoclinic.org/diseases conditions/malaria/diagnosistreatment/drc-20351190

${ }^{2}$ Disease Management. Final Academy of Managed Care PharmacyAvailable at http://www.amcp.org/WorkArea/DownloadAsset. aspx?id=9295Medical plants used in traditional treatment of malaria. Also available at academicjournals.org/article/ar.
}

confirmation of diagnosis with either microscopy or Rapid Diagnostic Test (RDT). Treatment based on clinical grounds should only be given if diagnostic testing is not immediately accessible within 2 hours of patients presenting for treatment. Drugs for treatment are prescribed based on the type of malaria parasite discovered, the severity of the infection, age and pregnancy statue. The antimalarial medicines recommended in the WHO model list of essential medicines $[10]^{3}$ for the curative treatment of malaria include; amodiaquine, artemether, artemether+lumefantrine, artesunate, artesunate+amodiaquine, chloroquine, mefloquine, sulfadiazine, pentamidine, etc.

\section{B. Alternative Medicine Diagnosis and Management Approach}

The use of traditional medicines for the treatment of malaria has been in existence for thousands of years and can be described as the origin of the two major classes (artemisinin and quinine derivatives) [11] of antimalarial drugs available today.

The major motivations to the use of traditional medicines today include increase in the number of drug resistance and the challenges associated with accessing of effective antimalarial services or drugs by people in the rural areas. Presently, more than one thousand plant species from over 100 families have been very useful in the treatment of malaria [12]. Some plants recommended for this purpose include: the use of Apple Cider Vinegar, Ginger, Cinnamon, Fever Nut, Orange Juice, Grapefruit, Citrus Limetta Fruit, Holy Basil, Alum, Herbal Teas, Chirayta, Datura, Fenugreek Seeds, Mustard Seed Oil and Turmeric.

\section{RELATED WORK}

In the study conducted by [13], an appraisal of ten potent African medicinal plants is presented. An up to date overview of ten potential medicinal plants from the African biodiversity which can be grouped under future phyto-pharmaceuticals for the treatment or management of several infectious and chronic diseases is carried out. Prominent scientific databases were explored to examine trends in the number of publications on the medicinal values of most African plants. A Decision Support System model for diagnosing tropical diseases using Fuzzy Logic is proposed in [14]. Results from the experiments carried out indicate that FL could be used to solve problems associated with data ambiguity, imprecision and even uncertainty. In [15], an algorithm for malaria diagnosis using fuzzy logic for treatment in Ghana is presented. A case study was conducted in Juaso District Government Hospital. MATLAB 7.8.0 was used for the design and simulation of the algorithm. A Fuzzy-Based System for the diagnosis and treatment of tuberculosis is presented in [3]. Mamdani inference method was used. The system was designed with Java, Microsoft Visio (2013), MySQL workbench, MySQL database, JSP and XHML. In [6] a survey of the medicinal plants used by traditional healers for the treatment of malaria in the Chipinge district in Zimbabwe is presented. The survey was undertaken to document how malaria is conceptualized

\footnotetext{
${ }^{3}$ WHO model list of essential medicines 20th list, 2017http://www.who.int/medicines/publications/essentialmedicines/en
} 
and diagnosed by traditional healers, and to record the medicinal plants used in the prevention and treatment of malaria, their mode of preparation and administration. In the work of [16] a documentation of herbal Medicines used for the treatment and management of human diseases by some communities in Southern Ghana is presented. The results of the study showed that herbal medicines are used for treatment and management of both common and specialized human diseases and that, factors of place and time are considered important during harvesting of plants for treatments.

A review of the existing related work revealed that there is a wide gap between alternative and pure medicine in the treatment of human diseases. Also, since both pure medicine and alternative medicine treatment methods are not without weaknesses or limitations, it becomes necessary to have a management approach housing these two major treatment methods.

\section{THE Proposed SySTEM}

The proposed system is a fuzzy based system based on the integration of both alternative and pure medicine for the management of malaria. Management in this context involves diagnosis, treatment and prevention of malaria. The intelligent of the system is derived from the result of an interactive decision process based on knowledge obtained from experts (medical doctors and alternative medicine practitioners) in the field.

The result of the diagnosis determines the next step in the management process to be taken. That is, prevention if malaria has not been diagnosed and treatment otherwise. Treatment here could be purely medical (drugs) or herbal or a combination of both when appropriate. Information in the patients' medical profile such as pregnancy status, age, presence of terminal diseases such as diabetes, ulcer etc. is also considered in the treatment process.

\section{A. The Proposed System Architecture}

System design shows the components that make up a system and also the relationship between them. Fuzzy based systems are made up of four major components; knowledge base, fuzzifier, inference engine and the defuzzifier. Some of these components are discussed in details. The architecture of the proposed system is presented in Fig. 1. It shows the connections existing between the system's components. The proposed integrated fuzzy based system consists of two major layers; the fuzzy layer and the management layer. Some of the sub-components of these layers are briefly described.

The fuzzy layer houses the fundamental components of a fuzzy based system. This is where fuzzy logic or rule is applied. As such, greater portion of the intelligent possessed by the system resides here. The components in this layer are discussed.

Fuzzifier: This component takes care of fuzzification. Fuzzification is the use of fuzzy membership function to change a real scalar value to a fuzzy value. Firstly, values within specified range are assigned variables or terms known as linguistic variables. The linguistic variables used in this work are; minor, moderate severe and very severe. A collection of the set of values that make up a linguistic variable is known as fuzzy set. The equation of the fuzzy set used in this work is expressed in (3), (4), (5) and (6).

$$
\begin{aligned}
& (X)=\left\{\begin{array}{cc}
0 & \text { if } x \leq 0.1 \\
\frac{x-0.1}{0.2} & \text { if } 0.1 \leq x \leq 0.3 \\
\frac{0.2-x}{0.1} & \text { if } 0.2 \leq x \leq 0.3 \\
0 & \text { if } x \quad \geq \quad 0.2
\end{array}\right. \\
& \mu \text { moderate }(x)=\left\{\begin{array}{cc}
0 & \text { if } x \leq 0.3 \\
\frac{x-0.3}{0.3} & \text { if } 0.3 \leq x \leq 0.6 \\
\frac{0.45-x}{0.15} & \text { if } 0.45 \leq x \leq 0.6 \\
0 & \text { if } x \quad \geq 0.45
\end{array}\right. \\
& \mu \text { severe }(x)=\left\{\begin{array}{cc}
0 & \text { if } x \leq 0.5 \\
\frac{x-0.6}{0.2} & \text { if } 0.6 \leq x \leq 0.8 \\
\frac{0.7-x}{0.1} & \text { if } 0.7 \leq x \leq 0.8 \\
0 & \text { if } x \geq 0.7
\end{array}\right.
\end{aligned}
$$

The development of rules which are used to determine the degree of membership is also part of the fuzzification process. A rule is fired whenever any of the precedence parameter evaluates to true. The first five rules used in this work are

\begin{tabular}{|c|c|c|c|c|c|c|c|c|c|c|c|c|}
\hline No & Fever & Headache & Nausea & Vomiting & Jaundice & $\begin{array}{l}\text { Enlarge } \\
\text { lever }\end{array}$ & $\begin{array}{l}\text { Joint } \\
\text { pain }\end{array}$ & $\begin{array}{l}\text { Body } \\
\text { weakness }\end{array}$ & Dizziness & $\begin{array}{l}\text { Loss of } \\
\text { appetite }\end{array}$ & MP & Conclusion \\
\hline 1. & $\mathrm{~m}$ & $\mathrm{~m}$ & $\mathrm{~m}$ & $\mathrm{~m}$ & $\mathrm{M}$ & $\mathrm{m}$ & $\mathrm{m}$ & $\mathrm{m}$ & $\mathrm{s}$ & $\mathrm{M}$ & $\mathrm{m}$ & $\mathrm{m}$ \\
\hline 2. & $\mathrm{mo}$ & $\mathrm{m}$ & $\mathrm{m}$ & $\mathrm{m}$ & $\mathrm{M}$ & $\mathrm{m}$ & $\mathrm{mo}$ & $\mathrm{mo}$ & $\mathrm{s}$ & $\mathrm{S}$ & mo & $\mathrm{mo}$ \\
\hline 3. & $\mathrm{~s}$ & $\mathrm{mo}$ & $\mathrm{m}$ & $\mathrm{m}$ & $\mathrm{M}$ & $\mathrm{m}$ & $\mathrm{m}$ & $\mathrm{s}$ & $\mathrm{s}$ & $\mathrm{S}$ & mo & $\mathrm{s}$ \\
\hline 4. & Vs & $\mathrm{m}$ & $\mathrm{m}$ & $\mathrm{m}$ & $\mathrm{M}$ & $\mathrm{m}$ & $\mathrm{s}$ & $\mathrm{s}$ & $\mathrm{m}$ & M & $\mathrm{s}$ & vs \\
\hline 5. & mo & $\mathrm{m}$ & $\mathrm{m}$ & mo & $\mathrm{M}$ & $\mathrm{m}$ & $\mathrm{mo}$ & mo & mo & $\mathrm{S}$ & mo & $\mathrm{mo}$ \\
\hline
\end{tabular}
given in Table I.

TABLE. I. FUZZY RULE BASE FOR DIAGNOSING MALARIA 


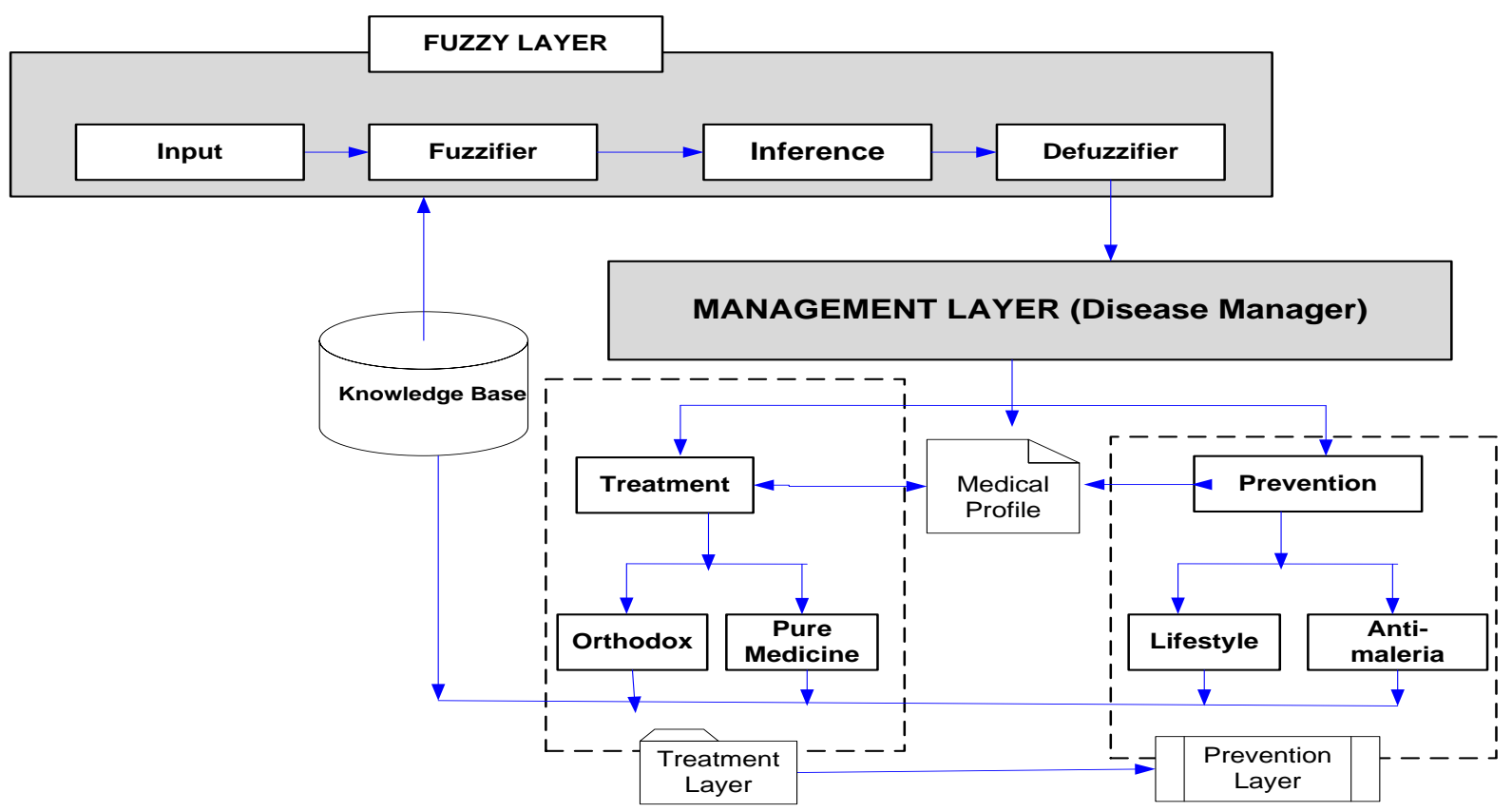

Fig. 1. Proposed System Architecture.

Deffuzzifier: It generates real values from fuzzy sets and corresponding degrees of membership. The output produced by the defuzzifier is known as crisp output. The center of gravity defuzzification method is used in this work. This is expressed in (6).

$C_{o} G=\frac{\sum \mu y\left(x_{i}\right) x_{i}}{\sum \mu y\left(x_{i}\right)}$

The Inference Engine: It processes the facts in the knowledge base to produce output for decision making. The intelligent of a fuzzy based system resides in the inference engine. It is modeled after the reasoning of experts in the field. The Root Sum Square (RSS) inference technique expressed in (7) is used in this work.

$\sqrt{\sum \mathrm{R}^{2}}=\sqrt{\left(\mathrm{R}_{1}^{2}+\right.} \mathrm{R}_{2}^{2}+\mathrm{R}_{3}^{2}+, \ldots, \mathrm{R}_{\mathrm{n}}^{2}$

Where: $R_{1}^{2}+R_{2}^{2}+R_{3}^{2}+\cdots+R_{n}^{2}$ are the strength values (truth values) of different rules which share the same conclusion. The Management Layer: Management at this phase excludes diagnosis because diagnosis is carried out at the fuzzy layer. The results derived from the fuzzy layer are channeled to the management layer for further action. The major components of this layer are; the treatment layer and the prevention lager.

This is as also presented in the proposed system architecture in Fig. 1.

The Treatment Layer: The treatment layer is further divided into two; pure medicine which administers or recommends pure medicine (drugs) for treatment and orthodox which recommends herbs for treatment. These two treatment methods may also be used complementarily when necessary. The choice of a particular treatment method depends on users' preferences, availability and suitability. The information in the patients' medical profile which include pregnancy statue, age, present of other illness, certain drugs and herbs is considered to ensure accuracy and safety in the administration of treatments.

The Prevention Layer: This takes care of the preventive measures which could be used to control the stop malaria infection. Preventive measures in the form of anti-malarial drugs and/or change in lifestyle are prescribed or recommended for people who have not yet been infected by malaria or people that have been offered treatment for malaria. Lifestyle changes include; the use of mosquito nets, personal hygiene etc.

\section{B. Experimental Setup and Results}

The proposed system is implemented in C\# in visual studio Integrated Development Environment. MySQL database is used for data storage. In the proposed system, adding a patient to the system activates the symptoms module shown in Fig. 2. The symptoms module shown in Fig. 3 determines the malaria statue of the patient based on symptoms such as fever, headache, etc. diagnosis based on symptoms alone could be misleading because the same set of symptoms may denote different medical conditions. To deal with this imprecision, a fuzzy analysis of the symptoms is performed by the fuzzification module shown in Fig. 4 Fuzzification is performed using the rules in the rule base given in Table I. This module determines the degree or the intensity of the identified symptoms as shown in Fig. 5. For the purpose of accuracy, the system is built to work with raw inputs i.e. symptoms and also medical laboratory test results. This figure shows the degree of each symptom based on medical laboratory test result supplied and a defuzzified or crisp value of the fuzzy value. The result derived from the fuzzy analysis and other conditions such as age and pregnancy statue is used to present appropriate management (prescription) and treatment method. Treatments which could be herbal as shown in Fig. 6 or medical presented in Fig. 7 or a combination of both are prescribed. Preventive 
measures as shown in Fig. 8 are also specified for all infected and non-infected patients. The result of this experiment shows that the proposed system can manage malaria. Management in this context includes diagnosis, treatment which is made up of pure medical treatment, alternative (herbal) treatment, complimentary treatment which is a combination of alternative (herbal) treatment method and pure medical treatment method.

\begin{tabular}{|c|c|c|}
\hline 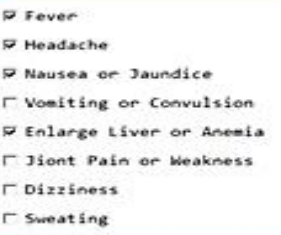 & $\begin{array}{l}r T \text { swollen breast } \\
r \text { Fatigue/vowiting } \\
\text { o slight bleeding } \\
\text { o mood swings } \\
r \text { Food Aversions } \\
\text { r constipation } \\
r \text { cravings }\end{array}$ & $\begin{array}{l}\Gamma \text { coughing 3-4uks } \\
0 \text { coughing blood } \\
r \text { chest pain } \\
o \text { Breathing pain } \\
r \text { weight loss } \\
\text { r wight sweat } \\
r \text { chills }\end{array}$ \\
\hline
\end{tabular}

Fig. 2. Symptom Module.

\section{Fuzzymal Test Result}

Malaria

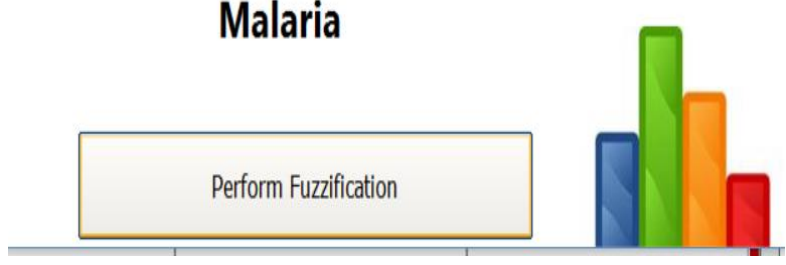

Fig. 3. Fuzzification Platform.

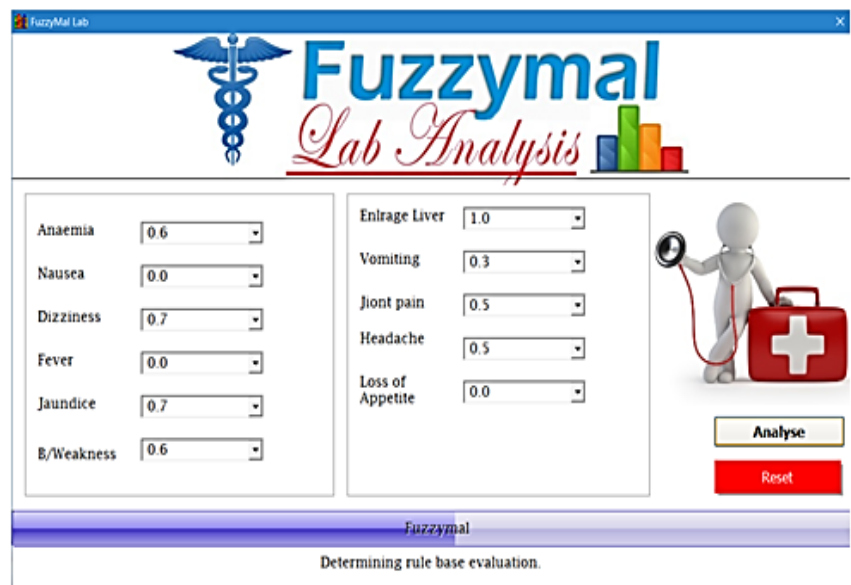

Fig. 4. Fuzzification Process.

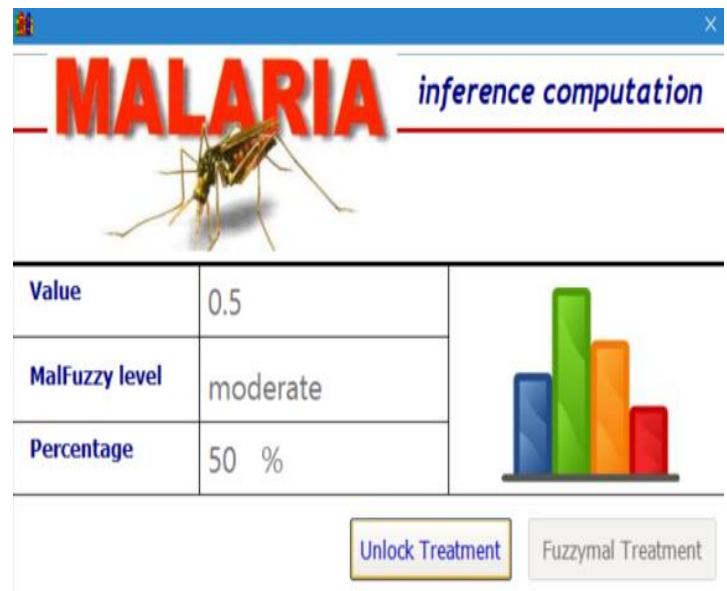

Fig. 5. Inference Computation.

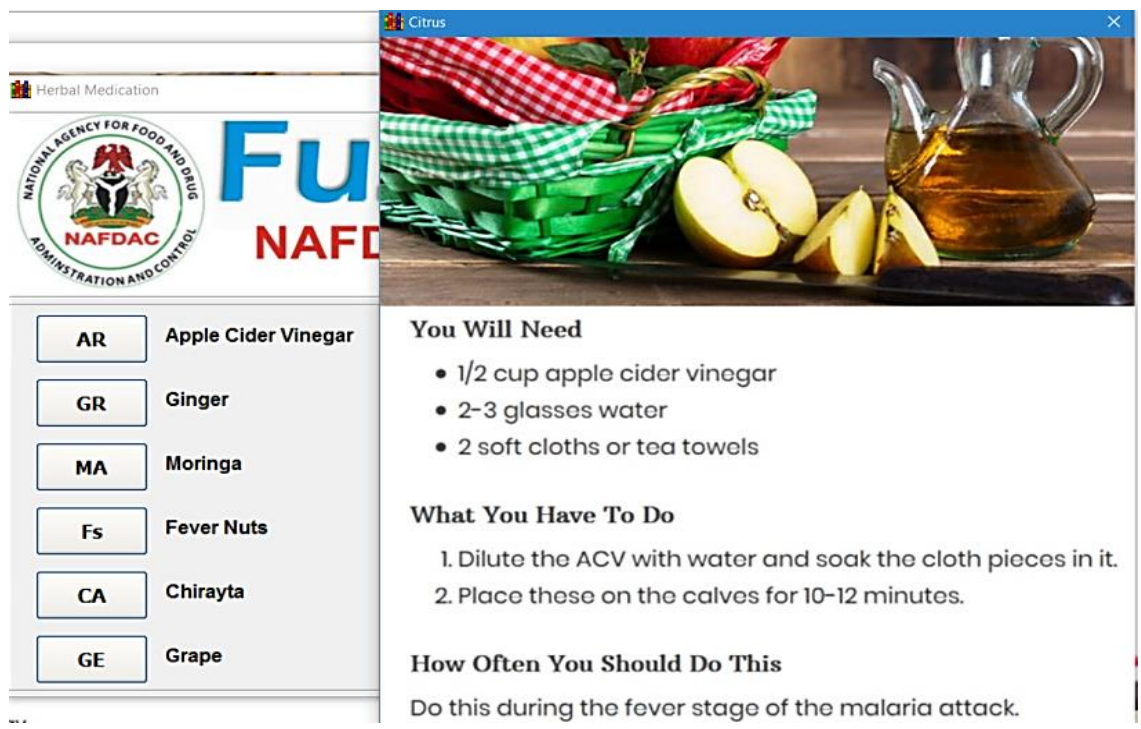

Fig. 6. Herbal (Prescribed) Treatment. 


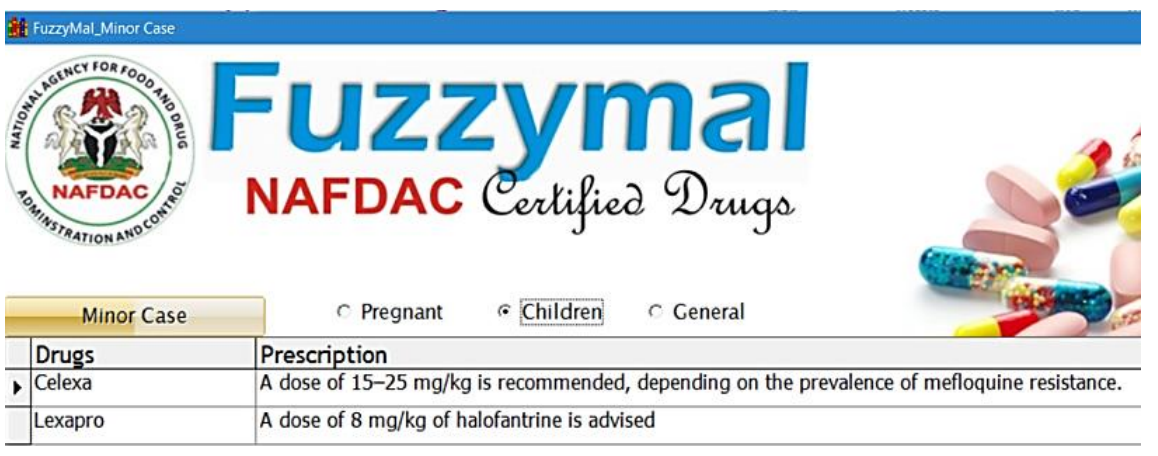

Fig. 7. Medical (Prescribed) Treatment.

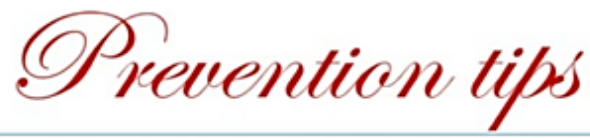

\begin{abstract}
1. Do not allow water to stagnate as this serves as the breeding ground for Anopheles mosquitoes, which reproduce and multiply, thus spreading the fever wherever they go.

2. Water bodies should be sprayed with mosquito repellent or other effective chemicals to keep the mosquito population under control.

3. Keep your surroundings clean by keeping your home clean, dry, and hygienic. Use disinfectants like Dettol, phenyl, etc. for cleaning your house and toilets.

4. Use mosquito repellents or mosquito coils while sleeping. If you are allergic to them, you can use mosquito nets to avoid being bitten by a mosquito. This will prevent their entry into your house.

5. Wear long-sleeved clothing and full-length pants while stepping out of the house.
\end{abstract}

Fig. 8. Malaria Prevention Tips.

\section{CONCLUSION AND RECOMMENDATIONS}

In conclusion, an integrated fuzzy based method which combines both orthodox (herbal) and pure medical treatment methods for diagnosis and management of human diseases (malaria) is proposed. With the result obtained it is clear that the proposed system can be used to manage malaria both medically and alternatively. Combining these two methods eliminates the weaknesses in the individual methods of management. However, the addition of complimentary treatment approach will be a reasonable improvement to the proposed method.

\section{CONTRIBUTIONS TO KNOWLEDGE}

This work presents the following contributions to knowledge:

- Development of an integrated fuzzy based system for the management of human diseases.

- This approach improves on the use of medical drugs or herbs for the treatment of diseases.

- The study establishes the possibility of using two treatment methods for the treatment of diseases.

\section{ACKNOWLEDGMENTS}

Our thanks go to the experts and reviewers who have contributed towards the development of this work.

\section{REFERENCES}

[1] J. Awotunde, O. Matiluko, and O. Fatai, "Medical diagnosis system using fuzzy logic", African Journal of Computing \& ICT. Vol. 7 No. 2, 99-106. 2014.

[2] Y. Djam, M. Gregory, H. Kimbi, and V. Nachamada, V. “A fuzzy expert system for the management of malaria. International Journal of Pure and Applied Sciences and Technology" Vol. 5 No.2, 84-108.2011.

[3] A. Angbera,M. Esiefarienrh, and I. Agaji, "Efficient fuzzy-based system for the diagnosis and treatment of tuberculosis", International Journal of Computer Applications, Technology and Research, Vol. 5, Issue 2, 2016.

[4] C. Chuen, "Fuzzy logic control system: Fuzzy logic controller -part I. IEEE Transaction on systems, man, and cybernetics", Vol.20, No.2, 404 $-418.2014$.

[5] G. William, "An optimization approach to employee scheduling using fuzzy logic". MSc. Thesis, California Polytechnic State University, San Luis Obispo). 2011.

[6] N. Talkmore, E. Charlott, A.Klooster, M. Jong,., and V. Jan (2015) "Medicinal plants used by traditional healers for the treatment of malaria in the Chipinge district in Zimbabwe". Journal of Ethno-pharmacology, Vol. 159, 224-237.

[7] Schrijvers G. (2009). Disease management: a proposal for a new definition. International journal of integrated care, 9, e06. 
[8] Malaria diagnosis and treatment-Mayo Clinic. Available at https://www.mayoclinic.org/diseases conditions/malaria/diagnosistreatment/drc-20351190.

[9] Disease Management. Final Academy of Managed Care Pharmacy. Available at http://www.amcp.org/WorkArea/DownloadAsset.aspx?id= 9295Medical plants used in traditional treatment of malaria. Also available at academicjournals.org/article/ar.

[10] WHO model list of essential medicines 20th list, 2017. http://www.who.int/medicines/publications/essentialmedicines/en

[11] Willcox, M. L., \& Bodeker, G. (2004). Traditional herbal medicines for malaria. BMJ (Clinical research ed.), 329(7475), 1156-1159

[12] Treatment and Management of Malaria Parasite. Available at http://www.malaria.com/questions/malaria-treatment-management
[13] F. Mahomoodly, "Traditional medicines in Africa: An appraisal of ten potent medicinal plants".Evidence-based complementary and alternative medicine. Vol. 2013, Article ID 617459. 2013.

[14] S. Olabiyisi, E. Omidiora, M. Olaniyan, and O. Derikoma, "Decision Support Model for Diagnosing Tropical Diseases Using Fuzzy Logic", Afr. J comp \& ICT Vol. 4. No. 2, 1-6, 2011.

[15] D. Quashie,K. Joseph, and B. James, "Designing algorithm for malaria diagnosis using fuzzy logic for treatment", International Journal of Computer Applications Vol. 91, No.17. 2014.

[16] A. Boadu and A. Asase, "Documentation of herbal medicine used for the treatment and management of human diseases by some communities in southern Ghana. Evidence Based Complementary and Alternative Medicines" Vol. 2017. 2017. 